\title{
Active Contours Guided by Echogenicity and Texture for Delineation of Thyroid Nodules in Ultrasound Images
}

\author{
Michalis A. Savelonas, Member, IEEE, Dimitris K. Iakovidis, Member, IEEE, Ioannis Legakis \\ and Dimitris Maroulis, Member, IEEE
}

\begin{abstract}
Thyroid nodules are solid or cystic lumps formed in the thyroid gland and may be caused by a variety of thyroid disorders. This paper presents a novel active contour model for precise delineation of thyroid nodules of various shapes according to their echogenicity and texture, as displayed in ultrasound (US) images. The proposed model, named JET (Joint Echogenicity-Texture), is based on a modified Mumford-Shah functional that, in addition to regional image intensity, incorporates statistical texture information encoded by feature distributions. The distributions are aggregated within the functional through new log-likelihood goodness-of-fit terms. The JET model requires only a rough region of interest within the thyroid gland as input and automatically proceeds with precise delineation of the nodules, revealing their shape and size. The performance of the JET model was validated on a range of US images displaying hypoechoic and isoechoic nodules of various shapes. The quantification of the results shows that the JET model: 1) provides precise delineations of thyroid nodules as compared to "ground truth" delineations obtained by experts, and 2) copes with the limitations of the previous thyroid US delineation approaches as it is capable of delineating thyroid nodules regardless of their echogenicity or shape.
\end{abstract}

Index Terms - Computer-Aided Diagnosis, Active Contours, Local Binary Patterns, Ultrasound, Thyroid Nodules.

\section{INTRODUCTION}

$\mathrm{U}$ LTRASOUND (US) is a widely used form of medical imaging, both as a primary modality and as an adjunct to other diagnostic procedures, providing substantial clues in differential diagnosis [1][2]. Its main advantages include non-invasiveness, low-cost and short acquisition times. The interpretation of US images is not a trivial task. A long learning curve is required for radiologists so as to acquire skills in recognizing the image features that comprise risk factors for different diseases, whereas it is difficult to remove the

This work was supported by the Greek General Secretariat of Research and Technology (25\%) and the European Social Fund (75\%), through the PENED 2003 program (grant no. 03-ED-662)

M.A. Savelonas, D.K. Iakovidis, and D. Maroulis are with Real-Time Systems \& Image Analysis Group, Dept. of Informatics and Telecommunications, Univ. of Athens, Panepistimiopolis, Illisia, 15784 Athens, Greece (e-mail: \{m.savelonas, d.iakovidis, d.maroulis\}@di.uoa.gr; phone: +30-210-7275307; fax: +30-210-7275333).

I. Legakis is with the Dept. of Endocrinology, Henry Dunant Hospital, Mesogion 107, 11526 Athens, Greece (e-mail: ilegak@med.uoa.gr). subjective element from the diagnostic process. A challenging task for US diagnostics is the assessment of thyroid nodules. Thyroid nodules are solid or cystic lumps formed in the thyroid gland. They may be caused by a variety of thyroid disorders and carry a considerable risk of malignancy. The most useful features of the US images, which are usually correlated with the pathology of the thyroid nodules, are echogenicity, texture and shape [3]. For example, recent studies [3][4] indicate that nodules of irregular boundary are associated with a higher malignancy risk. A precise US image delineation method capable of capturing these features or indicating their presence to the experts could contribute to the objectification of medical decisions, and could also be used as an educational tool for trainee radiologists.

Medical image segmentation approaches based on active contour models have been applied to images generated by medical imaging modalities as varied as US, magnetic resonance (MR), X-ray, computed tomography (CT) and angiography. Two-dimensional and three-dimensional active contours have been used to segment, visualize, track and quantify a variety of anatomic structures ranging in scale from the macroscopic to the microscopic. These structures include the heart, the cerebrum, a kidney, the lungs, objects such as brain tumors, a fetus, and even cellular structures such as neurons and chromosomes [5]. Cheng et al [6] developed and validated an automatic system using active contours for detecting the intimal and the adventitial layers so as to calculate the intima-media thickness of the common carotid artery. Plissiti et al [7] proposed an active contour model for the delineation of the lumen and media-adventitia border in sequential intravascular US frames. Chang et al [8] utilized a 3-D geodesic active contour to obtain the tumor contour for the pre- and the post-operative malignant breast excision by the vacuum assisted biopsy instrument Mammotome. Jeong et al [9] extended and combined the level set active contour segmentation approach and the agglomerative hierarchical k-means approach for unsupervised clustering. Their approach has been applied for the classification/differentiation of soft tissues in multiband high-resolution ultrasonic transmission tomography images.

This wide applicability of active contours can be attributed to the fact that these models consider an object boundary as a 
whole, unlike traditional low-level image processing methods such as edge detection and mathematical morphology, which merely consider local image information. Moreover, the inherent continuity and smoothness of active contours can compensate for noise, gaps and other irregularities in object boundaries. Furthermore, when formulated using level sets [10], active contours are able to adapt to topological changes such as splitting or merging, which is a requisite in cases of multiple pathological findings.

Most of the previous active contour applications on US image segmentation utilize information derived from image intensity to guide contour evolution. This is also the case with the variable background active contour model (VBAC) [11], proposed for the delineation of nodules on thyroid US images. VBAC copes with the presence of intensity inhomogeneity in thyroid US images, considering information from sparse background regions. However, its application is limited to hypoechoic or hyperechoic nodules, whereas the malignancy risk of isoechoic nodules is considerable as well [3].

The incorporation of textural information within the active contour framework is requisite in order to enable the delineation of US findings, which cannot be distinguished by their average intensity, as in the case of isoechoic thyroid nodules. The hybrid multi-scale method [12] involves a wavelet-based edge detection method and Hough transformation of the US images for the delineation of thyroid nodules regardless of their echogenicity. However, this method involves the classic instance of the Hough transform for circles detection which considers a-priori circular shapes for the nodules, thus limiting its practicality. Moreover, the edge detection procedure used makes the whole process sensitive to the presence of US noise. A method that would be capable of coping with the limitations of the afore mentioned approaches would constitute a practical tool for the computer-aided delineation of thyroid nodules. Towards this direction we have considered an integrated region-based image segmentation approach that co-evaluates the textural image features and guides the experts to identify the nodules. Aiming at an efficient and effective representation of texture we have considered the use of local binary pattern (LBP) distributions.

The LBP operator, introduced by Ojala et al [13], is defined so as to provide feature distributions offering a condensed encoding of local microstructures that capture textural information. It has been supported by various comparative studies on texture analysis [13]-[15] demonstrating that LBP texture representation can be superior to Gabor, wavelet and co-occurrence approaches, and presents a smaller computational overhead. Unlike the Gabor approach, which utilizes textural features calculated from the weighted mean of pixel values over a small neighborhood, the LBP operator considers each pixel in the neighborhood separately, providing even more fine-grained information. The feature distributions estimated using the LBP operator are invariant to any monotonic change in gray-level intensities, resulting in a robust representation of textures under varying illumination conditions and can be made multiscale and invariant against rotation [16]. These latter attributes of LBP texture representation are of particular importance in US image representation, since US images are acquired using various gain, frequency and magnification settings, as well as different transducer placements. Moreover, since feature distributions estimated using the LBP operator are not correlated with the absolute value of image intensity, they provide complementary information which can be effectively fused with it.

Recent applications utilizing the LBP feature distributions for medical image analysis include plaque segmentation of intravascular ultrasound [17], classification of melanocytic lesions [18], and automatic detection of gastrointestinal adenomas in video endoscopy [19]. Unsupervised texture segmentation algorithms utilizing the LBP feature distributions have been mainly based on hierarchical splitting and agglomerative merging [20], as well as on region-competition approaches [21].

Based on the above, we present JET (Joint Echogenicity-Texture), a novel active contour model that co-evaluates regional image intensity and LBP distributions by utilizing a modified Mumford-Shah functional. The dissimilarity of the regions inside and outside the active contour is assessed through new log-likelihood goodness-of-fit terms included within the functional. The JET model incorporates the capabilities of VBAC to provide delineations of hypoechoic and hyperechoic nodules, since these types of nodules are merely distinguishable by their differentiated echogenicity, and extends its capabilities providing delineations of isoechoic nodules. Moreover, it copes with the limitations of the hybrid multi-scale model by exploiting the topological adaptability of the level-sets used in its formulation.

The rest of this paper is organized in four sections. Section II briefly reviews the formulation of the LBP operator. The proposed JET model is presented in Section III. The results from its application for the delination of thyroid nodules in US images are apposed in Section IV and the conclusions of this study are summarized in Section V.

\section{LOCAL BINARY PATTERNS}

The LBP operator, as defined in [22], utilizes a binary representation of local texture patterns. Let $T$ be such a texture pattern, defined in a local neighborhood of a gray-level texture image as the joint distribution of the gray-levels of $P(P>1)$ image pixels:

$$
T=\tau\left(g, g_{0}, \ldots, g_{P-1}\right)
$$

where $g$ is the gray-level of the central pixel of the local neighborhood and $g_{P}(p=0, \ldots, P-1)$ represents the gray-level of $P$ equally spaced pixels arranged on a circle of radius $R(R>0)$, forming a circularly symmetric neighbor set.

Assuming that the differences $g_{p}-g$ are not affected by changes in mean luminance $g$, the joint difference distribution $\tau\left(g_{0}-g, \ldots, g_{P-1}-g\right)$ is invariant against gray-level shifts. Moreover, the LBP approach achieves invariance with respect 


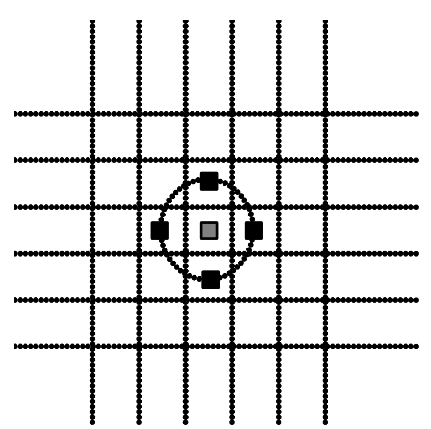

(a)

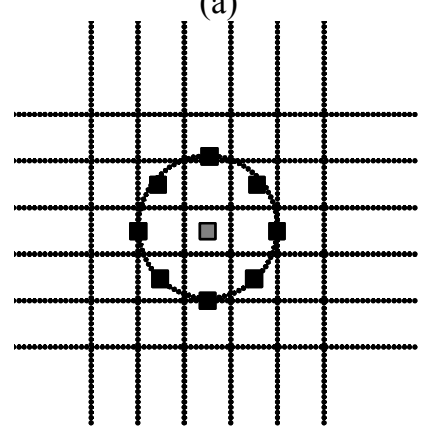

(c)

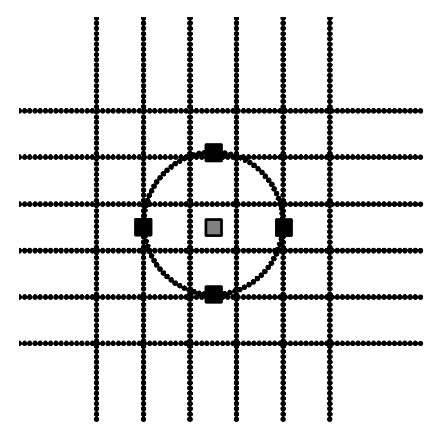

(b)

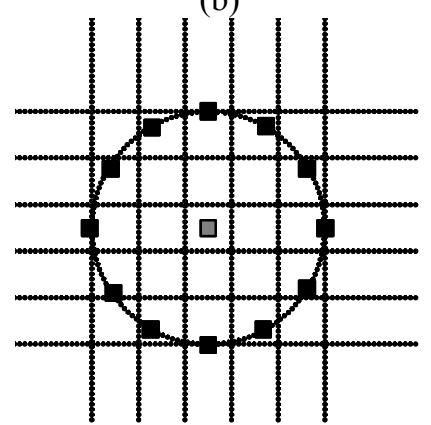

(d)
Fig. 1. Circularly symmetric neighbor sets for: (a) $P=4, R=1.0$, (b) $P=4, R=1.5$, (c) $P=8, R=1.5$, (d) $P=16, R=2.5$

to the scaling of the gray-levels by considering $H\left(g_{p}-g\right)$, instead of $g_{p}$-g, i.e. the joint signed difference distribution $T$ :

$$
T^{\prime}=\tau\left(H\left(g_{0}-g\right), \ldots, H\left(g_{P-1}-g\right)\right)
$$

where $H$ is the Heaviside function defined by:

$$
H(z)= \begin{cases}1, & \text { if } z \geq 0 \\ 0, & \text { if } z<0\end{cases}
$$

The LBP encoding is obtained by assigning a binomial factor $2^{p}$ to each term $H\left(g_{p}-g\right)$. A unique $\mathrm{LBP}_{P, R}$ value that encodes the spatial structure of the local image texture $T^{\prime}$ is estimated by:

$$
L B P_{P, R}=\sum_{p=0}^{P-1} H\left(g_{p}-g\right) 2^{p}
$$

The distribution of the $\operatorname{LBP}_{P, R}$ values calculated over an image region, comprises a highly discriminative feature vector for texture segmentation, as demonstrated in various comparative studies [13]-[15]. More detailed information concerning the LBP can be found in [15].

\section{THE JET MODEL}

We consider the reduced case of the Mumford-Shah problem, known as the minimal partition problem [23], which involves the minimization of the following functional:

$$
\begin{aligned}
F\left(C, \bar{c}_{+}, \bar{c}_{-}\right) & =\mu \cdot \operatorname{length}(C)+\int_{\text {inside }(C)} \frac{1}{b} \sum_{i=1}^{b} \lambda_{i}^{+}\left|u_{0}^{i}(x, y)-c_{+}^{i}\right|^{2} d x d y+ \\
& +\int_{\text {outside(C) }} \frac{1}{b} \sum_{i=1}^{b} \lambda_{i}^{-}\left|u_{0}^{i}(x, y)-c_{-}^{i}\right|^{2} d x d y
\end{aligned}
$$

where $u_{0}(x, y), i=1, \ldots, b$ are the components that describe the original image $u_{0}, C(s):[0,1] \rightarrow R^{2}$ is a parameterized curve, $c_{+}^{i}$ and $c_{-}^{i}, i=1,2, \ldots, b$ represent the average value of $u_{0}^{i}$ inside and outside the curve. The parameter $\mu$ weights the first, regularizing term of (5), whereas the parameters $\lambda_{i}^{+}$and $\lambda_{i}^{-}$, $i=1, \ldots, b$ weight the two fitting terms of (5). The regularizing term is associated with the shape of the curve and increases as the curve $C$ becomes irregular. The two fitting terms tend to decrease as the curve $C$ "fits" to homogeneous image regions. Each component $u_{0}^{i}(x, y), i=1, \ldots, b$, is defined over a single pixel $(x, y)$. The foreground and the background regions resulting from the segmentation of the image by the contour $C$, are denoted as "inside $C$ " and "outside $C$ ", respectively.

We incorporate the regional information encoded by means of LBP feature distributions into (5), by replacing the vector $u_{0}^{i}(x, y), i=1,2, \ldots, b$ which represents the image components at a single pixel $(x, y)$, with $D^{i}(x, y), i=1,2, \ldots, b$, where each component $D^{i}(x, y)$ corresponds to the $i$-th bin of the LBP distribution, and $b=2^{P}$ is the number of bins comprising each distribution. $D^{i}(x, y)$ encodes the textural properties of $k \times k$-pixel image regions centered at pixel $(x, y)$. This definition of $D^{i}(x, y)$ over an image region instead of a single pixel is based on the fact that texture is undefined at the single pixel level and it is always associated with an image region [24]. It should also be noted that, unlike features calculated at a single pixel level, $\operatorname{Di}(x, y)$ can be robust to the presence of small structures.

Moreover, motivated by [22] in which the log-likelihood statistic is suggested as an accurate similarity measure for LBP feature distributions, we consider the replacement of $\left|u_{0}^{i}(x, y)-c_{+}^{i}\right|^{2} \quad$ and $\quad\left|u_{0}^{i}(x, y)-c_{-}^{i}\right|^{2} \quad$ in (5), with $\left(1-D^{i}(x, y) \log \left(c_{+}^{i}\right)\right)$ and $\left(1-D^{i}(x, y) \log \left(c_{-}^{i}\right)\right)$ respectively. In addition, the intensity integrals of the scalar Chan-Vese model [25] are embedded in the formulation of the JET model, so as to maintain intensity information. These integrals are essential for the segmentation of thyroid US images containing hypoechoic or hyperechoic nodules, since they quantify the differences in average intensity between these types of nodules and their background. These considerations lead to the derivation of a new energy functional:

$$
\begin{aligned}
F^{\prime}\left(C, \bar{c}_{+}, \bar{c}_{-}\right)= & \mu \cdot \text { length }(C)+ \\
& +\int_{\text {inside }(C)} \frac{1}{b} \sum_{i=1}^{b} \lambda_{i}^{+}\left(1-D^{i}(x, y) \log \left(c_{+}^{i}\right)\right) d x d y+ \\
& +\int_{\text {outside }(C)} \frac{1}{b} \sum_{i=1}^{b} \lambda_{i}^{-}\left(1-D^{i}(x, y) \log \left(c_{-}^{i}\right)\right) d x d y+ \\
& +\lambda^{+} \int_{\text {inside( }(C)}\left(u_{0}(x, y)-c^{+}\right)^{2} d x d y+ \\
& +\lambda^{-} \int_{\text {outside }(C)}^{\int}\left(u_{0}(x, y)-c^{-}\right)^{2} d x d y
\end{aligned}
$$

where $u_{0}(x, y)$ is the original image, $c^{+}$and $c^{-}$represent the average intensity inside and outside the curve, and $\lambda^{+}$and $\lambda^{-}$ are weights for the image fitting terms. It should be noted that despite the fact that the VBAC model outperforms the scalar Chan-Vese model, it cannot be directly extended to incorporate textural information, because texture is a regional image property and VBAC considers a sparse approximately homogeneous background in which texture is indefinable.

The level set method [10] provides an efficient means for moving curves and surfaces, on a fixed regular grid, allowing for automatic topology changes, such as merging and splitting. Following [10], the curve $C$ is represented implicitly, by the 
zero level set of a Lipschitz function $\phi: \Omega \rightarrow R$, such that:

$$
\begin{aligned}
& C=\{(x, y) \in \Omega: \phi(x, y)=0\}, \\
& \text { inside }(C)=\{(x, y) \in \Omega: \phi(x, y)>0\}, \\
& \text { outside }(C)=\{(x, y) \in \Omega: \phi(x, y)<0\}
\end{aligned}
$$

Using the Heaviside function $H$ defined in (3) and the Dirac measure $d$, defined by:

$$
\delta(z)=\frac{d}{d z} H(z)
$$

where $z \in \mathrm{R}$, the level set formulation of the energy functional $F$, is:

$$
\begin{aligned}
& F^{\prime}\left(C, \bar{c}_{+}, \bar{c}_{-}\right)=\mu \cdot \int \delta(\phi(x, y))|\nabla \phi(x, y)| d x d y+\iint_{\Omega}\left[\frac{1}{b} \sum_{i=1}^{b} \lambda_{i}^{+}(1-\right. \\
& \left.D^{i}(x, y) \log \left(c_{+}^{i}\right)\right)+\lambda^{+}\left(\left(u_{0}(x, y)-c^{+}\right)^{2}\right] H\left(\phi(x, y) d x d y+\iint_{\Omega}\left[\frac{1}{b} \sum_{i=1}^{b} \lambda_{i}^{-}(1\right.\right. \\
& \left.-D^{i}(x, y) \log \left(c_{-}^{i}\right)\right)+\lambda^{-}\left(\left(u_{0}(x, y)-c^{-}\right)^{2}\right](1-H(\phi(x, y))) d x d y
\end{aligned}
$$

By keeping vectors $c_{+}^{i}$ and $c_{-}^{i}, i=1,2, \ldots, b$, as well as $c^{+}$and $c^{-}$ fixed, and minimizing $F^{\prime}$ with respect to $\phi$, the associated Euler-Langrange equation for $\phi$ is deduced:

$$
\begin{aligned}
\frac{\partial \phi}{\partial t} & =\delta(\phi)\left[\mu \cdot \operatorname{div}\left(\frac{\nabla \phi}{|\nabla \phi|}\right)-\frac{1}{b} \cdot \sum_{i=1}^{b} \lambda_{i}^{+}\left(1-D^{i} \log \left(c_{+}^{i}\right)\right)+\right. \\
& +\frac{1}{b} \cdot \sum_{i=1}^{b} \lambda_{i}^{-}\left(1-D^{i} \log \left(c_{-}^{i}\right)\right)+ \\
& \left.-\lambda^{+}\left(u_{0}-c^{+}\right)^{2}+\lambda^{-}\left(u_{0}-c^{-}\right)^{2}\right]
\end{aligned}
$$

where $t \in(0, \infty),(x, y) \in \Omega$ is an artificial time, which parameterizes the descent direction. The average values $c_{+}^{i}$ and $c_{-}^{i}, i=1,2, \ldots, b$, as well as $c^{+}$and $c^{-}$can be expressed with the use of $\phi$ by:

$$
\begin{array}{r}
c_{+}^{i}(\phi)=\frac{\int_{\Omega} D^{i}(x, y) H(\phi(x, y)) d x d y}{\int_{\Omega} H(\phi(x, y)) d x d y}, \\
c_{-}^{i}(\phi)=\frac{\int_{\Omega} D^{i}(x, y)(1-H(\phi(x, y))) d x d y}{\int_{\Omega}(1-H(\phi(x, y))) d x d y}
\end{array}
$$

and

$$
\begin{gathered}
c^{+}(\phi)=\frac{\int_{\Omega} u_{0}(x, y) H(\phi(x, y)) d x d y}{\int_{\Omega} H(\phi(x, y)) d x d y}, \\
c^{-}(\phi)=\frac{\int_{\Omega} u_{0}(x, y)(1-H(\phi(x, y))) d x d y}{\int_{\Omega}(1-H(\phi(x, y))) d x d y}
\end{gathered}
$$

A discretized and linearized version of (10) is:

$$
\begin{aligned}
\frac{\phi_{o, p}^{n+1}-\phi_{o, p}^{n}}{\Delta t}= & \delta\left(\phi_{o, p}^{n}\right) \cdot\left[\mu K-\frac{1}{b} \cdot \sum_{i=1}^{b} \lambda_{i}^{+}\left(1-D_{o, p}^{i} \log \left(c_{+}^{i}\right)\right)+\right. \\
& +\frac{1}{b} \cdot \sum_{i=1}^{b} \lambda_{i}^{-}\left(1-D_{o, p}^{i} \log \left(c_{-}^{i}\right)\right)- \\
& \left.-\lambda^{+}\left(u_{0, o, p}-c^{+}\left(\phi_{o, p}^{n}\right)\right)^{2}+\lambda^{-}\left(u_{0, o, p}-c^{-}\left(\phi_{o, p}^{n}\right)\right)^{2}\right]
\end{aligned}
$$

where $\Delta t$ is the time step, $\left(x_{o}, y_{p}\right)$ are the grid points for $1 \leq o, p \leq M, M$ represents the number of points on each side of the grid, $\phi_{o, p}^{n}=\phi\left(n \Delta t, x_{o}, y_{p}\right) \quad$ and $u_{0, o, p}=u_{0}\left(x_{o}, y_{p}\right)$ are approximations of $\phi(t, x, y)$ and $u_{0}(x, y)$ respectively, with $n \geq 0$, and $K$ represents the curvature of $\phi$, which is obtained from:

$$
K=\operatorname{div}\left(\frac{\nabla \phi}{|\nabla \phi|}\right)=\frac{\frac{\partial^{2} \phi}{\partial x^{2}} \cdot\left(\frac{\partial \phi}{\partial y}\right)^{2}-2 \frac{\partial \phi}{\partial x} \cdot \frac{\partial \phi}{\partial y} \cdot \frac{\partial^{2} \phi}{\partial x \partial y}+\frac{\partial^{2} \phi}{\partial y^{2}} \cdot\left(\frac{\partial \phi}{\partial x}\right)^{2}}{\left(\left(\frac{\partial \phi}{\partial x}\right)^{2}+\left(\frac{\partial \phi}{\partial y}\right)^{2}\right)^{\frac{3}{2}}}
$$

where $\partial \phi / \partial x, \partial \phi / \partial y$ are calculated by $\Delta^{x} \phi_{o, p} / \Delta s$, $\Delta^{y} \phi_{o, p} / \Delta s, \Delta^{x} \phi_{o, p}=\phi_{o+1, p}-\phi_{o, p}, \Delta^{y} \phi_{o, p}=\phi_{o, p+1}-\phi_{o, p}$ and $\Delta s$ is the step between two consecutive grid points of the discrete space.

In a practical implementation, a criterion should force the algorithm to stop when a stationary solution is reached. This criterion can be expressed as follows:

$$
Q \leq(\Delta t) \cdot(\Delta s)^{2}
$$

where:

$$
Q=\frac{\sum_{\phi_{o, p}^{n} \mid<\Delta s}\left|\phi_{o, p}^{n+1}-\phi_{o, p}^{n}\right|}{M^{\prime}}
$$

and $M^{\prime}$ is the number of grid points for which $\left|\phi_{o, p}^{m}\right|<\Delta s$.

The steps of the JET algorithm are summarized as follows:

1) Initialize $n \leftarrow 0, \phi^{0} \leftarrow \phi_{0}$

2) Calculate the $i$-th LBP histogram bin $D^{i}(x, y)$, for each $i, x, y$

3) Calculate the average values $c_{+}^{i}$ and $c_{-}^{i}$, for each $i$ by (10)

4) Calculate the average values $c^{+}$and $c^{-}$by (11)

5) Calculate the level set function $\phi^{n+1}$ by (13)

6) If inequality (15) is true then end

7) Set $n \leftarrow n+1$, repeat steps 2-6.

The application scenario of the JET algorithm requires that the expert provides a rough region of interest within the thyroid gland as input. Then, the contour specifying the region of interest evolves and, after a few iterations, the JET model converges to a final contour delineating the boundaries of one or more nodules within the thyroid gland. The segmentation quality obtained by the JET algorithm is practically invariant to the location of the initial contour. This is achieved, as in the case of the intensity-driven, scalar Chan-Vese algorithm, by utilizing the regularized heaviside function $\mathrm{H}$, suggested by Chan and Vese [25] to facilitate convergence to a global minimizer.

The JET algorithm has a complexity of $\mathrm{O}\left(n^{2}\right)$ for a $n \times n$ image, as it is the case with the simple intensity-driven scalar Chan-Vese algorithm. This is derived considering that the calculation of $D^{i}(x, y), i=1,2, \ldots, b$ for each pixel $(x, y)$ is performed only once and requires $\mathrm{O}\left(n^{2}\right)$ time, whereas each iteration of the algorithm involves the computation of $c^{+}, c^{-}$, $c_{+}^{i}$ and $c_{-}^{i}, i=1,2, \ldots, b$ in $\mathrm{O}\left(n^{2}\right)$ time as well.

\section{RESULTS}

Experiments were performed on 74 thyroid US images to investigate the performance of the JET model. The US images 
were acquired using a digital US imaging system HDI 3000 ATL with a 5-12 MHz linear transducer with a constant setting. The size of each US image of the dataset used was $256 \times 256$ pixels. The application framework of the JET model to US imaging is device dependent in the sense that a set of different parameter values is required for the segmentation of images acquired from different ultrasound devices. In most cases, parameter tuning requires technical skills and time-consuming manual interaction. This requirement can be overcome by employing a genetic algorithm (GA) approach, similar to the one introduced in [29] for the VBAC model. The GA was executed once for the device used in the experiments and a near optimal set of parameters was obtained. The ranges of the model parameters considered in the GA for the experiments performed in this study are $\lambda_{+(-)}^{i} \in\left[1.0 \cdot 10^{2}, 10.0 \cdot 10^{2}\right], \lambda^{+(-)} \in[0.1,10]$ and $\mu \in\left[0.5 \cdot 10^{1}, 50.0 \cdot 10^{2}\right]$, for $\lambda_{+}^{i}=\lambda_{-}^{i}, i=1,2, \ldots b$ and $\lambda^{+}=\lambda^{-}$, whereas the parameters on which the GA converged are $\lambda_{+}^{i}=\lambda_{-}^{i}=7.5 \cdot 10^{2}, i=1,2, \ldots b, \lambda^{+}=\lambda^{-}=1$ and $\mu=6.5 \cdot 10^{2}$. The values of the parameters $\lambda_{+}^{i}, \lambda_{-}^{i}, i=1,2, \ldots b$ were considered to be equal to each other in accordance with [25], as it is the case with $\lambda^{+}, \lambda^{-}$.

The parameters of the LBP operator used are $P=4$ and $R=1$, therefore $b=2^{4}=16$. The $\mathrm{LBP}_{4,1}$ distributions were selected as the most discriminative by a preliminary classification experiment, according to which balanced datasets of LBP distributions extracted from normal and nodular tissue samples, with $P=4,8,16$ and $R=1,2,4$, were classified with a nearest neighbors classifier employing the log-likelihood distance metric [22],[26]. A rule of thumb suggests that the number of entries for each bin of a histogram should be at least 10 . Considering that the $\mathrm{LBP}_{4,1}$ produces a 16-bin histogram, the number of entries required for the whole histogram is at least $16 \times 10=160$. Therefore $k=13$ corresponds to the minimum $k \times k$ neighborhood that satisfies this requirement $\left(13^{2}=169>160\right)$.

The active contour algorithm was implemented in Microsoft Visual $\mathrm{C}++$ and executed on a $3.2 \mathrm{GHz}$ Intel Pentium IV workstation. As delineation quality measures we have considered:

1) the overlap

$$
q(A, G)=\frac{A \cap G}{A \cup G}
$$

between the region $A$ delineated by the algorithm and the "ground truth" region $G$, obtained by following the rule that a pixel belongs to a nodule when it is included in at least two out of the three delineations drawn by experts [27].

2) the mean absolute distance

$$
e\left(A_{c}, G_{c}\right)=\frac{1}{2}\left\{\frac{1}{n} \sum_{i=1}^{n} d\left(a_{c}^{i}, G_{c}\right)+\frac{1}{m} \sum_{i=1}^{m} d\left(g_{c}^{i}, A_{c}\right)\right.
$$

between the two contours $A_{c}=\left\{a_{c}^{1}, a_{c}^{2}, \ldots, a_{c}^{n}\right\}$ and $G_{c}=\left\{g_{c}^{1}, g_{c}^{2}, \ldots, g_{c}^{m}\right\}$ of regions $A$ and $G$ respectively, in pixels. Distance $d$ is defined as

$$
d\left(a_{c}^{i}, G_{c}\right)=\min _{j}\left\|g_{c}^{j}-a_{c}^{i}\right\|
$$

3) the Dice coefficient [28]

$$
d c(A, G)=\frac{2 \times(A \cap G)}{|A|+|G|}
$$

where $|A|$ and $|G|$ are the numbers of pixels within the regions
$A$ and $G$.

The first two measures allow direct comparisons with the VBAC and the hybrid multi-scale models, which are evaluated in [11] and [12] using the overlap and the mean absolute distance, respectively.

A set of preliminary segmentation experiments has been performed on the available dataset so as to investigate the effect of parameter perturbations on the segmentation quality obtained by the JET model. Figure 2 illustrates the average values of $q$ along with the corresponding standard deviations obtained for perturbation ranges centered at the chosen parameters. It can be observed that the impact of the perturbations remains insignificant for a wide range of parameter values, for which the standard deviation of $q$ is overlapping. These ranges are $\lambda_{+(-)}^{i} \in\left[7.2 \cdot 10^{2}, 7.7 \cdot 10^{2}\right], i=1,2, \ldots b, \quad \lambda^{+(-)} \in[0.7,1.4]$, $\mu \in\left[2.5 \cdot 10^{2}, 10.5 \cdot 10^{2}\right]$. Similarly, slight variations of the device settings within the usual ranges applied in everyday clinical practice have a marginal effect on the segmentation quality obtained by the JET model, so it was preferred to use the initial GA-chosen set of parameter values for all the images of the dataset than to execute the GA for each image acquired with different device settings.

It is worth noting that the JET model does not require that the initial contour includes the nodule to be delineated, whereas the VBAC model subjects to this requirement because it involves calculations that are limited within the region defined by the initial contour. In order to investigate the degree to which the obtained segmentation quality is invariant to the location of the initial contour, as mentioned in Section III, we applied the JET model twice for each image; once with a contour surrounding the nodule and once at a random position within the region of the thyroid gland. The results showed a divergence of approximately $0.1 \%$ on average between the two delineations obtained per image. Figure 3 illustrates two initial contours that lead to practically identical delineations with an overlap $q$ of $90.4 \%$.

The experimental results are organized in three parts: the first and the second part present the performance of the JET model for the delineation of hypoechoic and isoechoic nodules respectively. The third part presents a comparative analysis of the JET model versus the VBAC and hybrid multi-scale models.

\section{A. Delineation of Hypoechoic Thyroid Nodules}

The values of $q, e$ and $d c$ obtained by the JET model for the cases of 38 thyroid US images featuring hypoechoic nodules are illustrated in Table I. The average $q, e$ and $d c$ obtained are $92.9 \pm 3.9 \%, 1.2 \pm 0.6$ pixels and $96.3 \pm 2.1 \%$ respectively. The minimum $q$ and $d c$ obtained are $79.8 \%$ and $89.0 \%$ in case 28 , whereas the maximum $e$ is 2.4 pixels in case 22. Moreover, $q$ exceeds $90 \%$ in 33 out of 38 cases, $d c$ exceeds $95 \%$ in 34 out of 38 cases, and $e$ is smaller than 2 pixels in 34 out of 38 cases.

Figure 4 illustrates the value of $q$ obtained by the JET model, as well as the ranges of $q$ obtained by each individual expert. The low ends of these ranges are lower than $90 \%$ in 19 out of 38 cases. This reflects a considerable interobserver variability, also addressed in [11],[12]. It can be observed that the JET model 
results in values of $q$ that fall within these ranges, in 30 out of 38 cases.

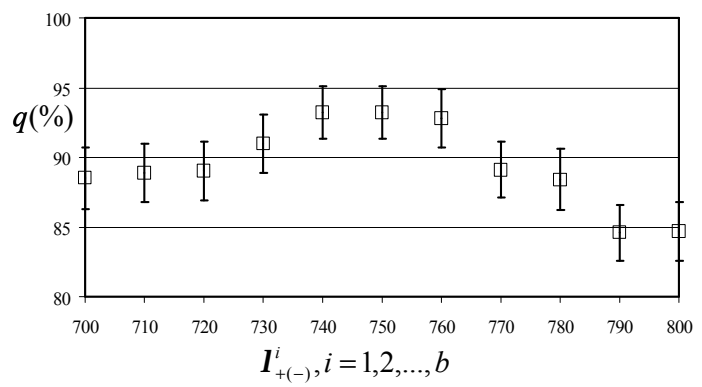

(a)

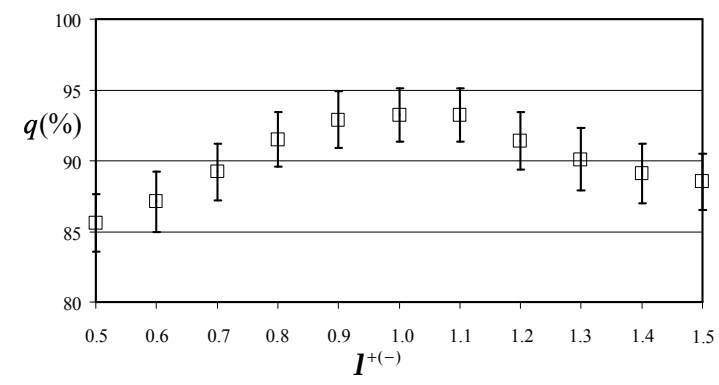

(b)

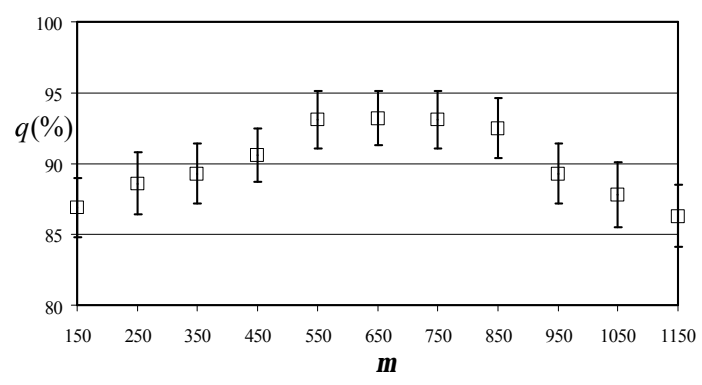

(c)

Fig. 2. Average $q$ obtained with the JET model over the available images for perturbations of: a) $\lambda_{+}^{i}=\lambda_{-}^{i}, i=1,2, . . b$ by keeping $\lambda^{+}=\lambda^{-}=1$ and $\mu=6.5 \cdot 10^{2} ;$ b) $\lambda^{+}=\lambda^{-}$by keeping $\lambda_{+}^{i}=\lambda_{-}^{i}=7.5 \cdot 10^{2}, i=1,2, \ldots b$ and $\mu=6.5 \cdot 10^{2} ;$ and c) $\mu$ by keeping $\lambda_{+}^{i}=\lambda_{-}^{i}=7.5 \cdot 10^{2}, i=1,2, \ldots b$ and $\lambda^{+}=\lambda^{-}=1$ The average $q$ is marked with squares.

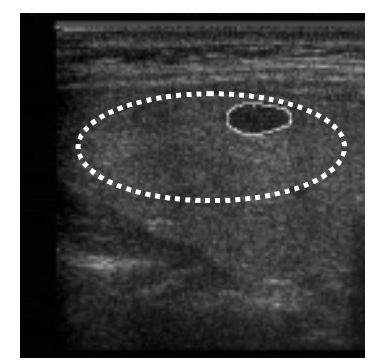

(a)

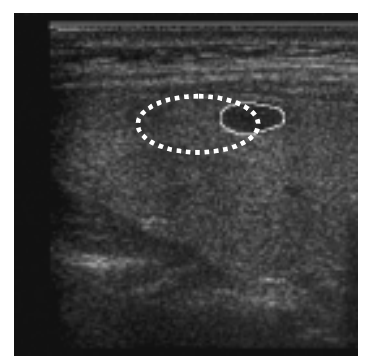

(b)
Fig. 3. Two delineations of a hypoechoic nodule, obtained by the JET model with different initial contours: (a) the initial contour includes the target nodule, and (b) the initial contour does not include the target nodule. The initial contours are indicated with dashed lines.
TABLE I

JET DELINEATION ACCURACY

\begin{tabular}{cccccccc}
\hline Case & \multicolumn{3}{c}{ JET } & & Case & \multicolumn{3}{c}{ JET } \\
\cline { 2 - 3 } \cline { 6 - 7 } & $q$ & $e$ & $d c$ & & $q$ & $e$ & $d c$ \\
\hline 1 & 94.1 & 0.8 & 97.0 & 20 & 94.7 & 0.6 & 97.3 \\
2 & 87.1 & 1.1 & 93.1 & 21 & 92.1 & 0.8 & 95.9 \\
3 & 90.4 & 1.1 & 95.0 & 22 & 91.4 & 2.4 & 95.5 \\
4 & 92.1 & 1.4 & 95.9 & 23 & 96.9 & 0.7 & 98.4 \\
5 & 85.9 & 2.2 & 92.4 & 24 & 93.9 & 0.6 & 96.9 \\
6 & 94.2 & 1.4 & 97.0 & 25 & 97.7 & 0.5 & 98.8 \\
7 & 95.9 & 0.8 & 97.9 & 26 & 93.5 & 1.9 & 96.6 \\
8 & 92.5 & 1.2 & 96.1 & 27 & 92.9 & 2.2 & 96.3 \\
9 & 95.6 & 0.8 & 97.8 & 28 & 79.8 & 1.5 & 89.0 \\
10 & 93.0 & 1.3 & 96.4 & 29 & 93.0 & 1.0 & 96.4 \\
11 & 93.4 & 1.5 & 96.6 & 30 & 93.5 & 1.3 & 96.6 \\
12 & 96.7 & 0.8 & 98.3 & 31 & 94.8 & 0.6 & 97.3 \\
13 & 92.7 & 0.4 & 96.2 & 32 & 83.5 & 1.4 & 91.0 \\
14 & 94.3 & 0.8 & 97.1 & 33 & 96.3 & 0.7 & 98.1 \\
15 & 94.6 & 1.2 & 97.2 & 34 & 92.6 & 1.6 & 96.2 \\
16 & 97.3 & 0.4 & 98.6 & 35 & 98.1 & 0.6 & 99.0 \\
17 & 92.8 & 1.0 & 96.3 & 36 & 94.2 & 1.8 & 97.0 \\
18 & 86.3 & 2.7 & 92.6 & 37 & 93.3 & 1.1 & 96.5 \\
19 & 95.9 & 0.7 & 97.9 & 38 & 93.1 & 0.8 & 96.4 \\
\hline
\end{tabular}

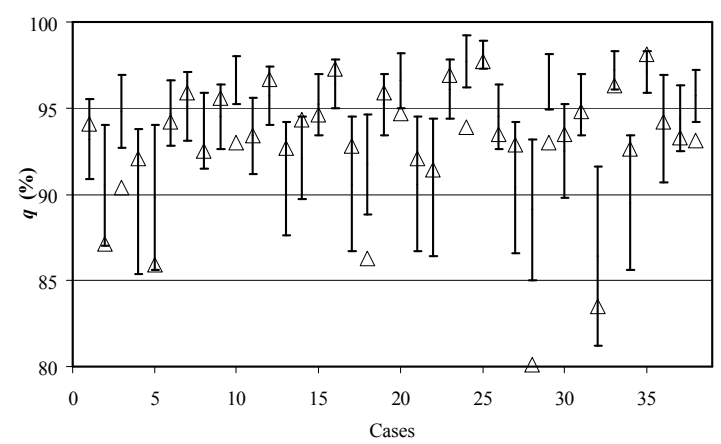

Fig. 4. Values of $q$ obtained with the JET model (triangles) and ranges obtained by individual experts (vertical bars), in cases of hypoechoic thyroid nodules.

Figure 5 illustrates delineations for indicative hypoechoic thyroid nodule cases, as drawn by JET models in comparison to the corresponding ground truth delineations.

\section{B. Delineation of Isoechoic Thyroid Nodules}

The values of $q, e$ and $d c$ obtained by the JET model are illustrated in Table II. The average $q, e$ and $d c$ obtained are $91.5 \pm 4.1 \%, 1.5 \pm 0.7$ pixels and $95.5 \pm 2.3 \%$. The minimum $q$ and $d c$ obtained are $75.3 \%$ and $85.9 \%$, whereas the maximum $e$ is 3.6 pixels, all in case 11 . Moreover, the value of $q$ exceeds $90 \%$ in 26 out of 36 cases, the value of $d c$ exceeds $95 \%$ in 24 out of 36 cases and the value of $e$ is smaller than 2 pixels in 28 out of 36 cases. It can be noticed that although the echogenicity of the isoechoic nodules is almost undifferentiated from the echogenicity of the normal thyroid parenchyma, the obtained delineation accuracy is marginally lower than the accuracy obtained for hypoechoic thyroid nodules. 

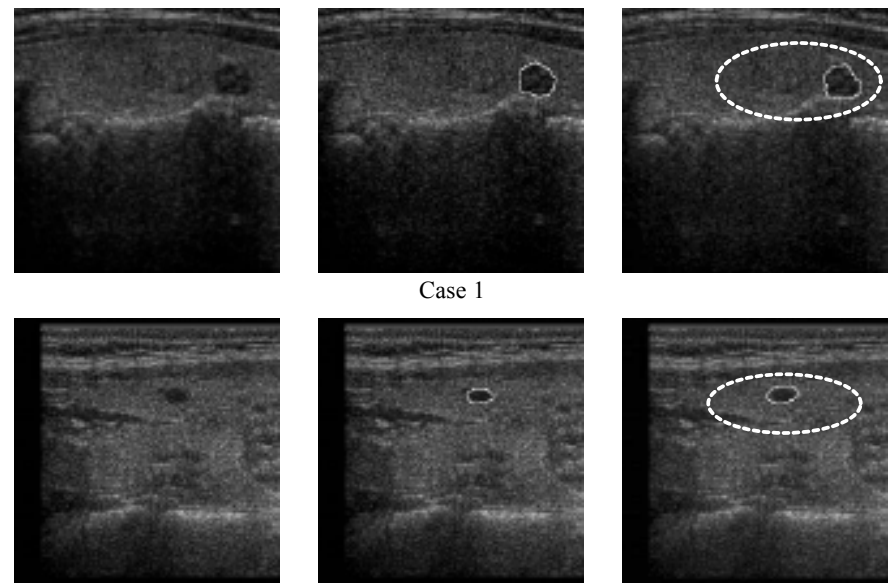

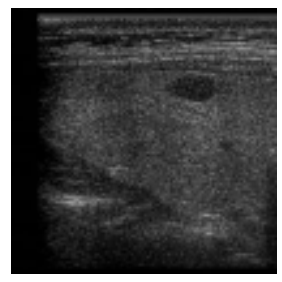

(a)

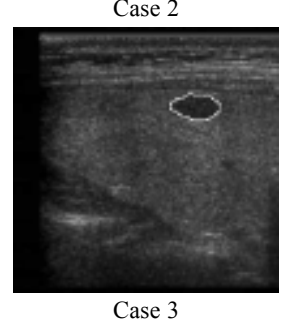

(b)

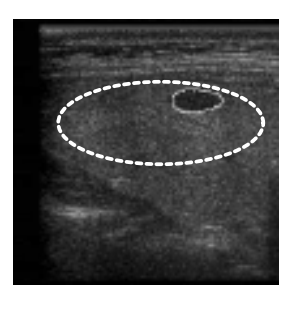

(c)
Fig. 5. Three thyroid US images with delineated hypoechoic nodules corresponding to the cases of Table 1: (a) original US images, (b) manual delineations, and (c) delineations obtained with the JET model. The initial contours are indicated with dashed lines.

Figure 6 illustrates the values of $q$ obtained by the JET model, as well as the ranges of $q$ obtained by each individual expert. It can be observed that the low end of $q$ obtained by the individual experts is lower than $90 \%$ in 19 out of 36 cases, whereas the JET model results in values of $q$ that fall within these ranges, in 22 out of 36 cases.

Figure 7 illustrates delineations of indicative isoechoic thyroid nodule cases, as drawn by the JET model in comparison to the corresponding ground truth delineations. The nodule that corresponds to the $11^{\text {th }}$ case is an isoechoic nodule that contains a cyst characterized by different intensity and texture than the rest of the nodule and caused JET to obtain the lowest $q$ in this part of experiments (Fig. 6).

\section{Comparison with previous thyroid US Delineation Models}

The application of the VBAC model on the dataset of hypoechoic thyroid nodules used in Section $A$ resulted in an average $q$ of $91.8 \pm 3.4 \%$ and an average $e$ of $2.7 \pm 1.2$ pixels. These results indicate that the delineation performance of the VBAC and JET models are comparable to each other for cases of hypoechoic thyroid nodules. However, the application of the VBAC model is limited to hypoechoic (and hyperechoic) cases, whereas the JET model is capable of segmenting isoechoic thyroid nodules as well.

In [12], the hybrid multi-scale method proposed was experimentally evaluated using delineations performed by two individual experts on a dataset of 40 thyroid US images comprising both, hypoechoic and isoechoic nodules. The reported results in terms of average $e$ measured over the whole dataset are $2.5 \pm 0.9$ pixels and $2.2 \pm 0.8$ pixels, based on delineations of two individual radiologists. The application of the JET model on the 74 images of the available dataset resulted in an average $e$ of $1.3 \pm 0.7$ pixels. A major drawback of the hybrid multi-scale method is that it requires a-priori information about the shape of the nodule boundaries to be detected, utilizing the classic instance of the generalized Hough transform which embeds the equation of a circle. This is very limiting for practical use as benign nodules most commonly have elliptical boundaries and malignant nodules exhibit boundary irregularities [3],[4]. Moreover, the hybrid-multi scale model is based on an edge detection scheme which makes the whole process sensitive to US noise. On the contrary, the JET model does not require any prior information about the shapes of the nodule boundaries, and the region-based formulation of the energy functional used contributes to noise-tolerant image segmentation.

The convergence time of the JET algorithm varies between 80 to 100 seconds depending on the image inhomogeneity and the location of the initial contour. This convergence time is comparable with the time required by the VBAC model [11], whereas in [12] the execution time of the hybrid multi-scale method is not referred.

\section{CONCLUSIONS}

In this paper, we proposed JET, a novel active contour model for the delineation of thyroid nodules in US images. The proposed model integrates regional image intensity and statistical textural feature distributions into a vector-valued, level-set active contour, capable of segmenting hypoechoic, isoechoic and hyperechoic nodules, the first two of which are associated with higher malignancy risk. The experimental evaluation on real thyroid US images lead to the following conclusions:

i) The JET model obtains precise delineations of thyroid nodules, regardless of their echogenicity. Its delineation performance is comparable to the one obtained by VBAC for cases of hypoechoic nodules, but it also extends the capabilities of VBAC, as it can delineate isoechoic nodules as well.

ii) The JET model copes with the limitations of the hybrid multi-scale model with respect to nodule shape by exploiting a topologically adaptive region-based approach.

iii) The delineation performance of the JET model is comparable to that of the experts, in both cases of hypoechoic and isoechoic nodules, suggesting that its application in everyday clinical practice is feasible.

iv) An added value to its applicability in practice is that the JET model does not require any training by the user. The only user input required is a rough region of interest within the thyroid gland, and the delineation is performed within a few seconds.

The JET model brings research one step closer to the objectification of the diagnostic process by utilizing explicit image features that encode more visual information about thyroid nodules. It can provide the diagnosticians with a second opinion on the delineation of a wider range of thyroid nodule types than state of the art methods. This is important especially 
in the case of follow-up diagnosis where the validity of conclusions drawn by the comparison of subsequent delineations depends on the delineation accuracy.

A limitation of the proposed model is that it is not always capable of distinguishing structures such as bigger blood vessels from actual nodules. However, such structures are not easily distinguishable even by expert radiologists, who usually need to employ additional color Doppler US imaging techniques in order to discriminate the two kinds of structures. In a similar fashion, the proposed model could be integrated into a future medical decision support system that would utilize color features from Doppler US images to achieve such discrimination.

TABLE II

JET DELINEATION ACCURACY

\begin{tabular}{|c|c|c|c|c|c|c|c|}
\hline \multirow[t]{2}{*}{ Case } & \multicolumn{3}{|c|}{ JET } & \multirow[t]{2}{*}{ Case } & \multicolumn{3}{|c|}{ JET } \\
\hline & $q$ & $e$ & $d c$ & & $q$ & $e$ & $d c$ \\
\hline 1 & 92.1 & 2.1 & 95.9 & 19 & 94.1 & 0.8 & 97.0 \\
\hline 2 & 89.6 & 1.8 & 94.5 & 20 & 96.1 & 1.2 & 98.0 \\
\hline 3 & 89.1 & 1.4 & 94.2 & 21 & 94.7 & 0.9 & 97.3 \\
\hline 4 & 94.5 & 0.8 & 97.2 & 22 & 92.5 & 1.3 & 96.1 \\
\hline 5 & 90.2 & 1.7 & 94.8 & 23 & 86.9 & 3.2 & 93.0 \\
\hline 6 & 91.3 & 0.9 & 95.5 & 24 & 93.7 & 1.1 & 96.7 \\
\hline 7 & 94.3 & 1.2 & 97.1 & 25 & 93.2 & 0.8 & 96.5 \\
\hline 8 & 87.7 & 1.0 & 93.4 & 26 & 88.9 & 2.0 & 94.1 \\
\hline 9 & 97.2 & 0.8 & 98.6 & 27 & 94.3 & 1.3 & 97.1 \\
\hline 10 & 87.4 & 2.5 & 93.3 & 28 & 94.2 & 0.7 & 97.0 \\
\hline 11 & 75.3 & 3.6 & 85.9 & 29 & 92.4 & 1.0 & 96.0 \\
\hline 12 & 86.6 & 2.6 & 92.8 & 30 & 95.0 & 1.3 & 97.4 \\
\hline 13 & 92.8 & 1.2 & 96.3 & 31 & 91.3 & 1.5 & 95.5 \\
\hline 14 & 95.9 & 0.5 & 97.9 & 32 & 88.4 & 1.4 & 93.8 \\
\hline 15 & 90.6 & 1.7 & 95.1 & 33 & 95.6 & 0.8 & 97.8 \\
\hline 16 & 93.5 & 0.7 & 96.6 & 34 & 91.9 & 1.6 & 95.8 \\
\hline 17 & 86.4 & 2.0 & 92.7 & 35 & 95.3 & 0.9 & 97.6 \\
\hline 18 & 91.2 & 1.9 & 95.4 & 36 & 90.2 & 2.3 & 94.8 \\
\hline
\end{tabular}

Future perspectives of this work include:

i) Derivation of additional information from video frame sequences for the identification of thyroid nodules.

ii) Malignancy risk assessment of the delineated nodules utilizing quantitative indexes.

iii) Embedment of the JET model into an integrated medical decision support system combining heterogeneous information from various sources for identification and automated assessment of thyroid nodules.

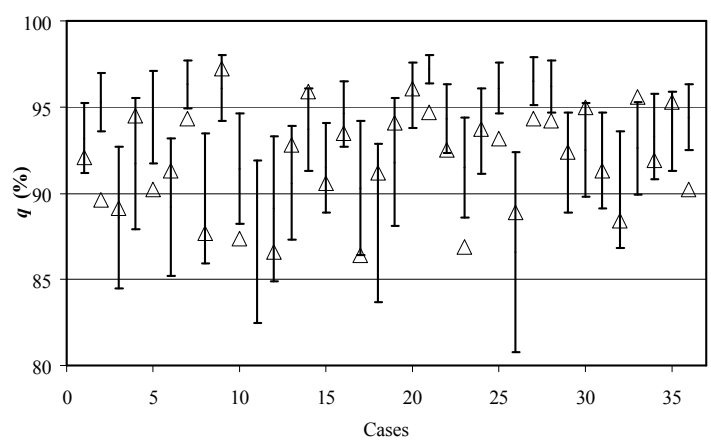

Fig. 6. Values of $q$ obtained with the JET model (triangles) and ranges obtained by individual experts (vertical bars), in cases of isoechoic thyroid nodules. In case 11 , JET resulted in an overlap of $75.3 \%$.
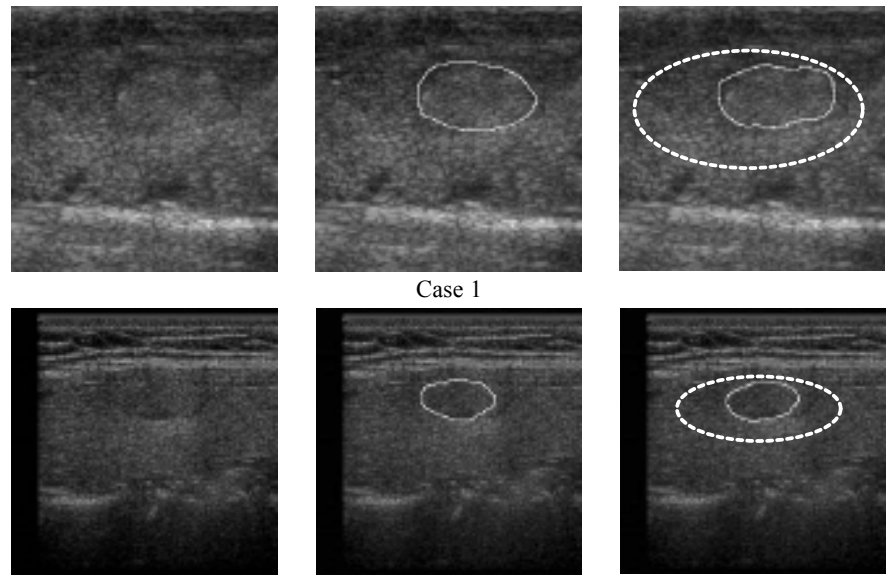

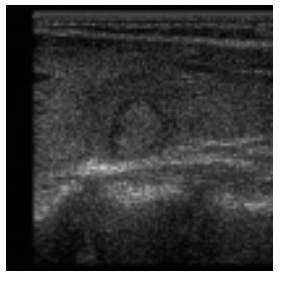

(a)

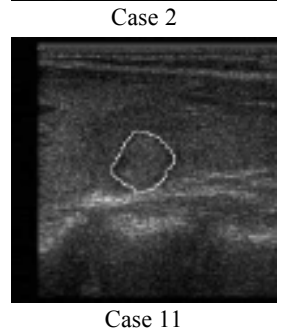

(b)

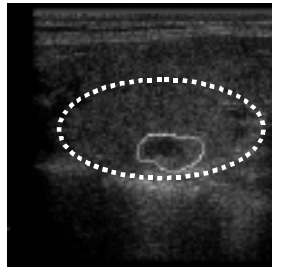

(c)
Fig. 7 Three thyroid US images with delineated isoechoic nodules corresponding to cases of Table 2: (a) original US images, (b) manual delineations, and (c) delineations obtained with the JET model. The initial contours are indicated with dashed lines.

\section{ACKNOWLEDGMENT}

We would like to thank very warmly Dr. N. Dimitropoulos, M.D., radiologist, for his kind and invaluable cooperation in this work. We gratefully acknowledge the time he spent for the assessment of the thyroid nodules and the medical feedback he provided so generously. Moreover, we would like to thank the Dept. of Radiology of EUROMEDICA S.A. and the Depts. of Radiology and Endocrinology of the General Hospital of Athens, Henry Dunant, for the provision of high quality anonymized data as well as for their assistance in the assessment of the thyroid nodules.

\section{REFERENCES}

[1] H.K. Ching et al, "Stepwise Logistic Regression Analysis of Tumor Contour Features for Breast Ultrasound Diagnosis," Proc. IEEE Ultrasonics Symposium, vol. 2, Atlanta, GA, USA, 2001, pp. 1303-1306.

[2] H.W. Müller, S. Schröder, C. Schneider, and G. Seifert, "Sonographic Tissue Characterization in Thyroid Gland Diagnosis-A Correlation between Sonography and Histology," Klinische Wochenschrift, vol. 63, pp. 706-710, 1985.

[3] E. Papini et al, "Risk of Malignancy in Nonpalpable Thyroid Nodules: Predictive Value of Ultrasound and Color-Doppler Features," Journal of Clinical Endocrinology and Metabolism, vol. 87, no. 5, pp. 1941-1946, 2002.

[4] J.R. Wienke, W.K. Chong, J.R. Fielding, K.H. Zou, C.A. Mittelstaedt, "Sonographic Features of Benign Thyroid Nodules: Interobserver Reliability and Overlap with Malignancy," J. Ultrasound Med., vol. 22, no. 10, pp. 1027-1031, 2003.

[5] T. McInerney and D. Terzopoulos, "Deformable Models in Medical Image Analysis: A Survey," Medical Image Analysis, vol. 1, no. 2, 1996.

[6] D.C. Cheng, X. Jiang, A. Schmidt-Truckass, and K.S. Cheng, "Automatic Intima-Media Thickness Measurement of Carotid Artery Wall in B-mode 
Sonographic Images," Proc.IEEE Int. Symp. Biomedical Imaging, 2006, pp. 912-915.

[7] M.E. Plissiti, D.I. Fotiadis, L.K. Michalis, G.E. Bozios, "An Automated Method for Lumen and Media-Adventitia Border Detection in a Sequence of IVUS Frames," IEEE Trans. On Inf. Tec. in Biomedicine, vol. 8, no. 2, pp. 131-141, 2004.

[8] R.F. Chang, W.J. Wu, C.C. Tseng, D.R. Chen, W.K. Moon, “ 3-D Snake for US in Margin Evaluation for Malignant Breast Tumor Excision Using Mammotome," IEEE Trans. On Inf. Tech. in Biomedicine, vol. 7, no. 3, pp. 197-201, 2003

[9] J.W. Jeong, D.C. Shin, S. Do, V.Z. Marmarelis, "Segmentation Methodology for Automated Classification and Differentiation of Soft Tissues in Multiband Images of High-Resolution Ultrasonic Transmission Tomography," IEEE Trans. Med. Imaging, vol. 25, no. 8, pp. 1068-1078, 2006.

[10] S. Osher, J. Sethian, "Fronts Propagating with Curvature-Dependent Speed: Algorithms Based on the Hamilton-Jacobi Formulations," Journal of Computational Physics, vol. 79, pp.12-49, 1988.

[11] D.E. Maroulis, M.A. Savelonas, D.K. Iakovidis, S.A. Karkanis, N. Dimitropoulos, "Variable Background Active Contour Model for Computer-Aided Delineation of Nodules in Thyroid Ultrasound Images," IEEE Trans. Inf. Tech. in Biomedicine, vol. 11, no. 5, pp. 537-543, 2007.

[12] S. Tsantis, N. Dimitropoulos, D. Cavouras, and G. Nikiforidis, "A Hybrid Multi-Scale Model for Thyroid Nodule Boundary Detection on Ultrasound Images," Computer Methods and Programs in Biomedicine, vol. 84, pp. 86-98, 2006.

[13] T. Ojala, M. Pietikäinen, and D. Harwood, "A Comparative Study of Texture Measures with Classification based on Feature Distributions," Pattern Recognition, vol. 29, pp. 51-59, 1996.

[14] P. Paclic, R. Duin, G.V. Kempen, and R. Kohlus, "Supervised Segmentation of Textures in Backscatter Images," Proc. of IEEE International Conference on Pattern Recognition, vol. 2, 2002, pp. 490-493.

[15] T. Mäenpää, and M. Pietikäinen, "Classification with color and texture: Jointly or separately?" Pattern Recognition, vol. 37, no. 8, pp. 1629-1640, 2004.

[16] T. Mäenpää, "The Local Binary Pattern Approach to Texture Analysis-Extensions and Applications," PhD dissertation, Dept. of Elec. Inf. Eng., Oulou University, Finland, 2003.

[17] O. Pujol and P. Radeva, "Supervised texture classification for intravascular tissue characterization," Handbook of Biomedical Image Analysis, eds. J.S. Suri, C. Yuan, D. Wilson, S. Laximinarayan, vol. 2: Segmentation Models part B, Springer, 2005

[18] J. Kontinen, J Röning, and R. Mackie, "Texture Features in the classification of Melanocytic Lesions," Proc. Int. Conf. on Image Analysis and Processing (ICIAP), vol. 2, Florence, Italy, 1997, pp. 453-460.

[19] P. Wang, S.M. Krishnan, C. Kugean, and M.P. Tjoa, "Classification of endoscopic images based on texture and neural network," Proc. Int. Conf. on Engineering in Medicine and Biology (EMBS), vol. 4, Istanbul, Turkey, 2001, pp. 3691-3695.

[20] T. Ojala and M. Pietikäinen, "Unsupervised texture segmentation using feature distributions," Pattern Recognition, vol. 32, no. 3, pp. 477-486, 1999.

[21] Q. Xu, J. Yang, and S. Ding, "Texture Segmentation using LBP embedded Region Competition," Electronic Letters on Computer Vision and Image Analysis, vol. 5, no. 1, pp. 41-47, 2005.

[22] T. Ojala, M. Pietikäinen, and T. Mäenpää, "Multiresolution Gray-Scale and Rotation Invariant Texture Classification with Local Binary Patterns," IEEE Trans. on Pattern Analysis and Machine Intelligence, vol. 24, no. 7, pp. 971-987 2002.

[23] D. Mumford, and J. Shah, "Optimal Approximation by Piecewise Smooth Functions and Associated Variational Problems," Commun.Pure Appl. Math., vol.42, pp 577-685, 1989.

[24] M. Unser, and M. Eden, "Nonlinear Operators for Improving Texture Segmentation Based on Features Extracted by Spatial Filtering," IEEE Trans. Systems, Man and Cybernetics, vol. 20, no. 4, pp. 804-815, 1990.

[25] T.F. Chan, L.A. Vese, "Active Contours Without Edges," IEEE Trans. Image Processing, vol. 7, pp. 266-277, 2001.

[26] S. Theodoridis, K. Koutroumbas, Pattern Recognition, $3^{\text {rd }}$ edition, Academic Press, 2006.
[27] M.R. Kaus, S.K. Warfield, F.A. Jolesz, R. Kikinis, "Segmentation of Meningiomas and Low Grade Gliomas in MRI," Proc. Int. Conf. on Medical Image Computing and Computer-Assisted Intervention, Cambridge, England, 1999, pp. 1-10.

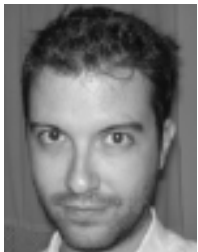

Michalis A. Savelonas (M'08) received the B.Sc. degree in Physics in 1998, the M.Sc. degree in Cybernetics in 2001 with honors, and the Ph.D. degree in the area of Medical Image Analysis in 2008 with honors, all from the University of Athens, Greece. Dr. Savelonas has co-authored 18 papers on biomedical applications and image analysis. Currently, he is a Research Fellow in the Dept. of Informatics and Telecommunications, University of Athens, Greece. His research interests include image analysis, segmentation, pattern recognition and biomedical systems.

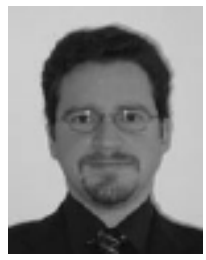

Dimitris K. Iakovidis (M'05) was born in Athens in 1973. He received his B.Sc. degree in Physics from the University of Athens, Greece. In April 2001, he received his M.Sc. degree in Cybernetics with honors and in February 2004 his Ph.D. degree from the Dept. of Informatics and Telecommunications, University of Athens, Greece.

He has been working for over ten years in the field of medical informatics and has been collaborating with many European hospitals and health centers as an expert on biomedical systems. Currently, among other academic positions, he holds a senior researcher position at the University of Athens, Greece.

Dr. Iakovidis has co-authored more than 60 research papers and book chapters, and he is a reviewer in 12 international journals, including IEEE Trans. on Image Processing, and IEEE Trans. Biomedical Engineering. He has been actively involved in more than 10 European and National R\&D projects. His research interests include image processing and analysis, data mining, pattern recognition with applications on biomedical systems and bioinformatics.

Ioannis Legakis obtained his B.Sc. in Medicine and his Ph.D. in Endocrinology, in 1991 and 1993, respectively, from the University of Athens, Greece. Currently he is with Henry Dunant hospital. Moreover, he is a Research Fellow in the Dept. of Medicine, and in the Dept. of Informatics and Telecommunications, University of Athens, Greece.

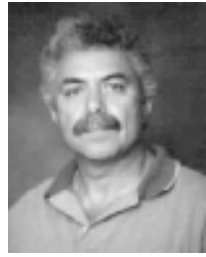

Dimitris Maroulis (M'02) received the B.Sc. degree in Physics, the M.Sc. degree in Radioelectricity, the M.Sc. in Cybernetics and the Ph.D. degree in Informatics, all from the University of Athens, Greece, in 1973, 1977, 1980 and 1990, respectively. Currently, he is an Associate Professor in the Dept. of Informatics of the University of Athens.

He has been working in the above Dept. in various teaching and research activities, including projects with the European Community. He has co-authored more than 90 articles in scientific journals and conferences on data acquisition systems, real time systems, image analysis and biomedical applications. His research interests include biomedical, data acquisition, real-time, image/signal processing systems and bioinformatics. 